\title{
COMPREENDENDO O SIGNIFICADO DE QUALIDADE DE VIDA SEGUNDO IDOSOS PORTADORES DE DIABETES MELLITUS TIPO II
}

\author{
Understanding the meaning of quality of life to older persons with type-2 diabetes \\ mellitus
}

\section{Comprendiendo el significado de la calidad de vida según ancianos portadores de diabetes mellitus tipo II}

\section{RESUMO}

Trata-se de uma pesquisa qualitativa com abordagem fenomenológica, objetivando descrever o significado de qualidade de vida, segundo relatos de idosos portadores de diabetes mellitus tipo II, e avaliar as repercussões da doença sobre sua vida. Entrevistamos 12 idosos diabéticos, no período de setembro a outubro de 2008, entre um e quarenta anos de evolução da doença. Foi feita a seguinte questão norteadora: "Para o Sr. (a), o que significa qualidade de vida?" A análise dos discursos mostrou facetas relevantes ligadas ao cotidiano do idoso com diabetes mellitus. Para eles, a qualidade de vida está intimamente relacionada à saúde física, independência na vida diária e econômica, integração social, supor te familiar e saúde mental-espiritual. A restrição alimentar foi o ponto de maior repercussão do diabetes sobre seu modo de viver. Verificou-se que cabe aos profissionais de saúde ampliar o diálogo profissional-paciente, promovendo autonomia e independência no cuidado e corresponsabilização.

Palavras-chave: Idoso. Diabetes mellitus. Qualidade de Vida.

\begin{abstract}
This is a qualitative study with a phenomenological approach, aiming to describe the meaning of quality of life based on statements given by elderly with type-2 diabetes mellitus and evaluate the disease effects on their lives. Twelve older persons who had been suffering from type-2 diabetes mellitus from one to forty years were interviewed from September to October 2008. The following guiding question was asked: "What does quality of life mean to you?" Discourse analysis showed relevant facets of the daily lives of older persons with diabetes mellitus. In their view, quality of life is closely related to good physical health, independence in daily and financial life, social integration, family support and good mental and spiritual health. Dietary restriction was the aspect mostly affecting their lifestyles. It was observed that health care professionals must enhance professional-patient dialogue, thus promoting autonomy and independence in care provision and co-responsibilization.
\end{abstract}

Keywords: Elderly. Diabetes Mellitus. Quality of Life.

\section{Resumen}

Esta es una investigación cualitativa con enfoque fenomenológico que pretende describir el significado de la calidad de vida según relatos de ancianos portadores de diabetes mellitus tipo Il y evaluar las repercusiones de la enfermedad sobre su vida. Entrevistamos a 12 ancianos diabéticos en el período de septiembre a octubre de 2008, entre uno y cuarenta años de evolución de la enfermedad. Se hizo la siguiente cuestión guía: "Para usted, ¿qué significa calidad de vida?" El análisis de los discursos mostró facetas relevantes ligadas al cotidiano del anciano con diabetes mellitus. Para ellos, la calidad de vida está íntimamente relacionada con la salud física, independencia en la vida diaria y económica, integración social, soporte familiar y salud mental y espiritual. La restricción alimentar ha sido el punto de mayor repercusión de la diabetes sobre su modo de vivir. Se observó que compete a los profesionales de salud ampliar el diálogo profesional-paciente, promoviendo autonomía e independencia en el cuidado y corresponsabilización.

Palabras clave: Anciano. Diabetes mellitas. Calidad de Vida.

"Enfermeira. Aluna regularmente matriculada no Curso de Especialização em Saúde da Família da Faculdade de Medicina de Botucatu da Universidade Estadual Paulista "Júlio de Mesquita Filho" - UNESP. Botucatu - SP. Brasil. E-mail: jp_enf@hotmail.com,²Enfermeira. Aluna regularmente matriculada no Curso de Especialização em Saúde da Família da Faculdade de Medicina de Botucatu da Universidade Estadual Paulista "Júlio de Mesquita Filho" - UNESP. Botucatu-SP. Brasil. E-mail: suelenalvesrocha@gmail.com, ${ }^{3}$ Enfermeira. Profa. Dra. do Departamento de Enfermagem da Faculdade de Medicina de Botucatu da Universidade Estadual Paulista Paulista "Júlio de Mesquita Filho" - UNESP. Botucatu-SP. Brasil. E-mail: rpopim@fmb.unesp.br 


\section{INTRODUÇÃO}

0 crescente aumento da população idosa é hoje um fenômeno universal. Nos países desenvolvidos, este processo se deu de forma lenta e associado às melhorias nas condições gerais de vida. Por outro lado, nos países em desenvolvimento, ele acontece de forma acelerada, havendo uma grande necessidade de políticas sociais e de saúde adequadas para atender às novas demandas emergentes. ${ }^{1}$

Segundo o Ministério da Saúde (MS), entre 1950 e 2025, a população idosa no país crescerá 16 vezes, ao passo que a população total crescerá cinco vezes, inserindo nosso país na sexta colocação em contingente de idosos no mundo com 34 milhões de pessoas. ${ }^{2}$

No contexto do envelhecimento populacional brasileiro, associado ao aumento da expectativa de vida, configura-se o quadro de transição epidemiológica, expresso pela diminuição de doenças transmissiveis e consequente incremento das crônicas não transmissiveis.

Entre as doenças crônicas não transmissíveis, o diabetes mellitus (DM) aparece como importante causa de morbimortalidade, principalmente entre os idosos. ${ }^{3}$ É definido como uma anormalidade endócrino-metabólica, caracterizada por uma deficiência absoluta do hormônio insulina ou relativa quando sua secreção pelo pâncreas é defasada e/ou por uma ação insuficiente do hormônio nos tecidos alvos. ${ }^{4}$

0 Diabetes Mellitus (DM), pela sua etiologia, apresenta-se em dois tipos mais frequentes, são o tipo l ell. 0 tipo I relaciona-se a uma deficiência absoluta na secreção do hormônio insulina, resultante de uma destruição autoimune das células-betas do pâncreas, compreendendo cerca de 5\% a $10 \%$ do total de casos. 0 tipo II é uma combinação de resistência à ação do hormônio e resposta secretora inadequada de insulina compensatória, compreendendo $90 \%$ a $95 \%$ do total de casos. ${ }^{4}$

Segundo estimativas da Organização Mundial da Saúde (OMS), o número de portadores de DM em todo mundo era cerca de 177 milhões em 2000, com expectativa de alcançar 350 milhões em 2025. No Brasil, em 2006 era aproximadamente 6 milhões, devendo alcançar 10 milhões em $2010 .^{5}$

Na pessoa idosa, a forma mais frequente é o Diabetes Mellitus tipo II (DM2). A hiperglicemia, manifestação principal, ocasiona lesões em diversos órgãos e sistemas do organismo, principalmente coração, olhos, rins e sistema nervoso. A dislepidemia, muitas vezes associada, pode desencadear problemas cardiovasculares como hipertensão, aterosclerose, angina e infarto do miocárdio, fatores que influem na funcionalidade física, psicológica e social do portador, prejudicando sua adaptação e vida produtiva. ${ }^{6}$

Em estudo realizado em uma cidade do interior paulista, para a população de idosos estudada, não ser portador de DM foi associado à satisfação com a vida. ${ }^{7}$ Isso revela que, no imaginário coletivo, o DM é uma doença temida, tem evolução insidiosa e traz consequentes alterações ao longo da vida cotidiana das pessoas.

Entende-se por qualidade de vida, segundo a OMS, "a percepção do indivíduo acerca de sua posição na vida, de acordo com o seu contexto cultural e sistema de valores nos quais vive e em relação aos seus objetivos, expectativas, padrões e preocupações". Trata-se, portanto, de um conceito marcado pela subjetividade e multidimensionalidade, uma vez que envolve os componentes essenciais da condição humana, sejam eles físicos, psicológicos, sociais, culturais ou espirituais. ${ }^{8}$

Em geral, a análise da qualidade de vida dos indivíduos tem, nos últimos anos, se consolidado como importante variável, tanto para a prática clínica como para a pesquisa científica na área da saúde, uma vez que permite melhor avaliar e estimar efeitos de intervenções, bem como seu impacto no bem-estar físico, psicológico e social dos pacientes. ${ }^{8}$

Assim, levando-se em consideração a complexidade dos problemas inerentes à vivência da cronicidade, particularmente agravados pelo processo de envelhecimento, pretende-se, nesse estudo, compreender o significado de qualidade de vida, segundo as experiências relatadas por idosos portadores de DM2.

\section{METODOLOGIA}

Nesse estudo, de natureza qualitativa, optou-se pela fenomenologia social de Alfred Schütz. A trajetória da pesquisa fenomenológica social busca chegar à essência do fenômeno interrogado com vistas à sua compreensão. Nesta busca, é fundamental a questão da subjetividade, na medida em que a fenomenologia se propõe, enquanto ciência da experiência vivida, a descrever essa experiência. ${ }^{9}$

Desta forma, a questão do significado no referencial fenomenológico é entendida na perspectiva do que é atribuído pelo sujeito que vive a experiência, conforme o fenômeno se mostra aos seus olhos, considerando que suas ações são direcionadas segundo seus motivos, permeadas pelos seus relacionamentos interpessoais. ${ }^{10}$

Desse modo, o referencial fenomenológico busca 0 significado da experiência vivida, contribuindo na busca da compreensão do homem, sujeito do cuidado, e vem, portanto, ao encontro dos propósitos desta pesquisa.

A região de inquérito foi constituída pela qualidade de vida relatada enquanto portador de DM tipo II.

A saturação teórica deu-se com 12 sujeitos, sendo critério de inclusão ser idoso (60 anos ou mais), portador de DM tipo II, com um mínimo de um ano de evolução da doença e atendido no ambulatório de geriatria e gerontologia do Centro de Saúde Escola, da Faculdade de Medicina de Botucatu UNESP.

Vale ressaltar que a coleta de dados foi realizada após a obtenção de parecer favorável do Comitê de Ética em Pesquisa, 
Idosos portadores de diabetes: qualidade de vida

conforme ofício 258/2008-CEP-Botucatu e a autorização dos atores que participaram deste estudo por meio da assinatura do Termo de Consentimento Livre e Esclarecido, respondendo a questão norteadora: "Para o Sr.(a), o que significa qualidade de vida?". Com anuência do sujeito, a entrevista foi gravada, transcrita na íntegra e, posteriormente, deletada.

A análise e interpretação dos dados seguiram os passos propostos por Parga Nina ${ }^{11}$ :

- Leitura demorada e atenta dos depoimentos, enquanto material não estruturado, visando apreender os significados relatados pelos sujeitos;

- Identificação das categorias temáticas que vão se apresentando, a partir dos discursos, as quais comportem os significados de qualidade de vida para o idoso portador DM2, bem como possíveis repercussões da doença em seu modo de vida;
- Releiturados depoimentospara extração e registro de trechos dos discursos quetiverem aspectos afins dos significados que compõem as categorias anteriormente identificadas;

- Síntese das unidades de significado para chegar à estrutura do fenômeno e à sua essência.

\section{RESULTADOS E DISCUSSÃO}

As convergências das unidades de significado dos discursos desvelaram a estrutura do fenômeno interrogado, sendo possível a construção de quatro categorias temáticas que expressam a essência do significado de qualidade de vida para este grupo de idosos diabéticos.

A Figura 1 sintetiza os temas desvelados:

Figura I - Diagrama dos significados de qualidade de vida para o idoso portador de Diabetes Melitus tipo II

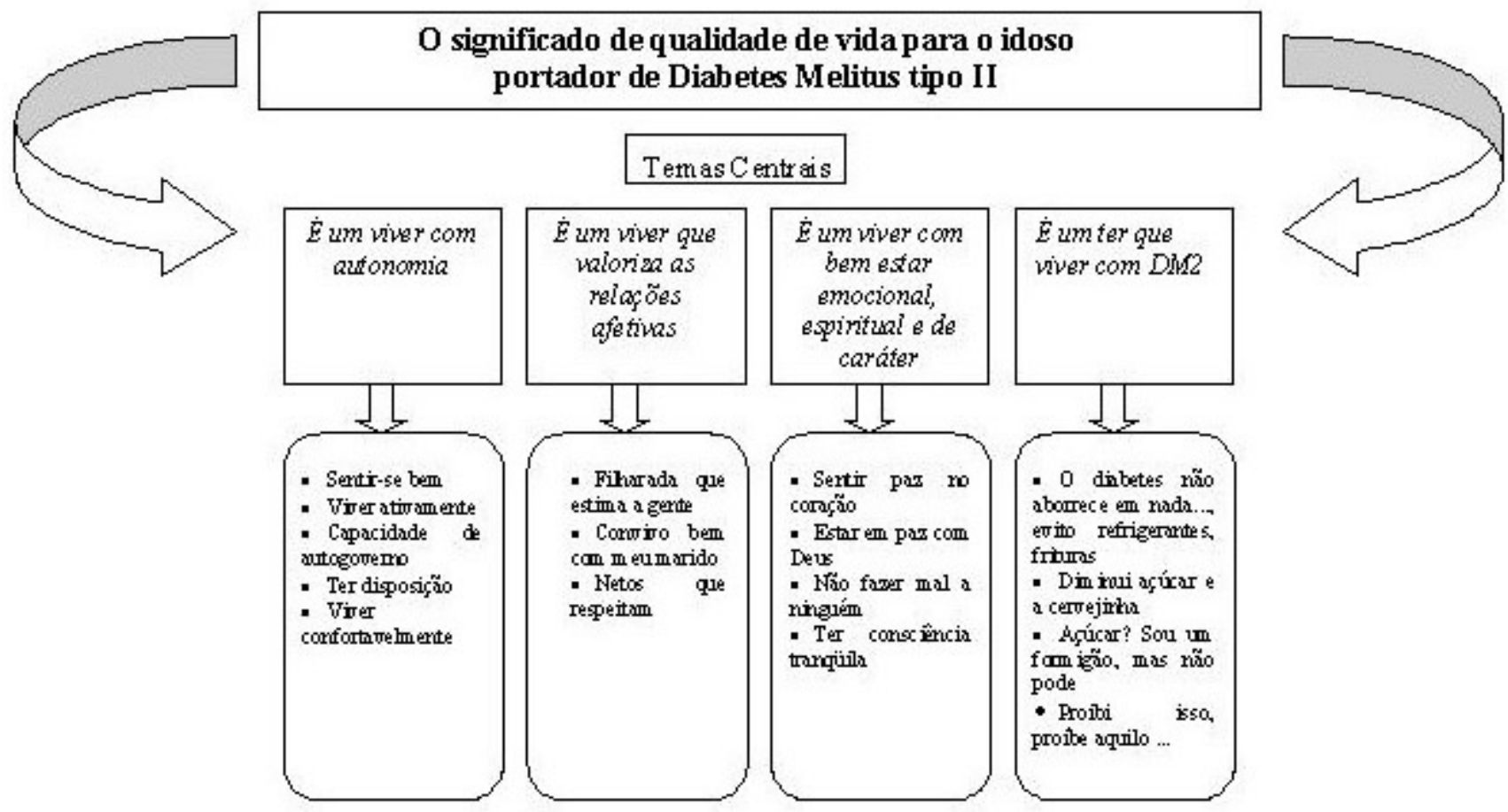

\section{É viver com autonomia}

Para este tema, convergiram as falas dos idosos que consideram que qualidade de vidaé sentir-se bem, viver ativamente com saúde e disposição para realizar suas atividades diárias e ter a capacidade de determinar suas próprias escolhas.

Qualidade de vida pra mim é você ter bastante saúde, ter disposição. (S7) Pra mim qualidade de vida é você ter disposição, não ficar naquela moleza, isso eu tenho graças a Deus [...]. (S11) Qualidade de vida é a gente viver bem, estar num lugar tranquilo, poder descansar e ter uma vida boa, eu acho que qualidade de vida é a gente se sentir bem. (S12)
Percebe-se que o dizer "sentir-se bem" está intimamente associado ao bem-estar físico, ou seja, para estes idosos manter-se independente fisicamenteé primordial. Logo, quando não possuem boa funcionalidade física, sentem-se desprovidos de qualidade de vida, conforme evidenciam os relatos abaixo:

Qualidade de vida é você poder sair, andar e ter disposição para fazer as coisas, isso é bom, é uma coisa maravilhosa [...] Mas eu não tenho essa disposição, estou muito gorda. (S2) Depois que fiquei velha fico plantada dentro de casa, não enxergo direito, quebreia bacia, andar como? Enjoa ficar parada, vou passar uma linha na agulha e não consigo, nem andar no quintal ando mais [...]. (S3) 
Para o idoso, a perda ou diminuição da capacidade funcional e de autonomia às vezes são mais importantes que a própria questão de morbidade, visto que estas estão diretamente relacionadas à qualidade de vida. ${ }^{8}$

Por meio de alguns discursos, percebe-se que este grupo de idosos valoriza a possibilidade de ter em mãos o controle de suas próprias decisões e vontades e que este fato contribui para um viver com mais qualidade.

\section{[...] Énão interferir na vida de ninguém e não deixar} que interfiram na minha. (S8) Faço meu servicinho, lavo minha roupa, faço minha comidinha [...] fico ali [...]. Não tenho quem me ajude, mas também não tenho quem me aborreça, não gosto de empregada, elas mudam as coisas de lugar e depois eu não acho. (S4)

A preservação da autonomia física e cognitiva, para o idoso, o poder de ir e vir, de tomar decisões, garantem-lhe um envelhecimento bem-sucedido, garantem-lhe uma postura ativa perante a vida e a sociedade. Isso confirmado, afasta-se da condição de envelhecer com fragilidade, em que há vulnerabilidade com maior suscetibilidade às doenças $e$ instalaç̃es de síndromes geradoras de dependência. ${ }^{12}$

Identificou-se, ainda, nos depoimentos, a importância da autonomia financeira que traz consigo a garantia de conforto material e, consequentemente, maior tranquilidade durante a terceira idade. Afinal, não possuir recursos financeiros suficientes pode ser um fator de estresse e preocupação.

Gasto aquilo que está no meu alcance, para não andarem batendo palma cobrando lá em casa. (S5) Ter uma vida boa para mim seria ter uma boa condição financeira, porque procuro fazer regime e tudo, mas a gente não tem as coisas adequadas pra comer, né? (S10) Vida boa pra mim seria que nem rico, não ter que fazer nada em casa. (S11) Éviver confortavelmente, num ambiente sadio. (S6)

Em estudo realizado em Uberaba com pacientes diabéticos, a questão financeira foi citada como um dos fatores que geram mais dificuldade de adesão ao tratamento do DM, uma vez que este requer uma mudança nos hábitos de vida, principalmente na aquisição de alimentos saudáveis. ${ }^{13}$

Outra pesquisa realizada em Botucatu esclarece que os idosos, ponderando a satisfação com a vida, consideram o conforto domiciliar um fator essencial, lembrando que os fatores socioeconômicos têm influência importante na qualidade de vida, já que estes oferecem suporte para o bem-estar do indivíduo. Diferentemente desta pesquisa, nosso estudo com o idoso diabético aponta para uma maior valorização do bemestar físico e cognitivo, embora o confor to domiciliar tenha sido citado.
Nos depoimentos, observou-se ainda que um dos fatores que influi sobre o viver saudável é o fato de poder realizar alguma atividade específica, um trabalho, por exemplo. Ao possuir uma ocupação, o idoso mantém um vínculo externo, com compromissos e outras pessoas, que lhes dão a sensação de produtividade, de independência, de autonomia. Portanto, manter-se produtivo e ativo influencia positivamente para um viver saudável na terceira idade.

Para os idosos diabéticos, ter autonomia física, cognitiva e financeira contribui para um envelhecer com qualidade de vida.

\section{É viver valorizando os relacionamentos afetivos}

Segundo Schütz, é por meio do relacionamento e da comunicação que vamos experimentando e sedimentando experiências, as quais nos diferenciam uns dos outros, nos orientam em nossas condutas, em nossos medos e receios, alegrias, em nossos motivos. É na comunicação com os outros que podemos existir, no sentido de nos mostrarmos e de perceber o outro. E é nessa troca de subjetividades que expressamos nossos sentimentos. 0 afeto é fundamental para perceber e compreender o outro. Ele pode nos motivar a tomar decisões, a aprender o novo, a perdoar. ${ }^{9}$

A família e as pessoas próximas são influências expressivas para um viver mais saudável, pois a convivência social lhes oferece um suporte.

Pra mim o que tem de bom na minha vida é a filharada que estima, cuida da gente. (S3) 0 principal é a gente se sentir bem onde a gente está, onde a gente mora, com quem a gente mora, isso é importante! Graças a Deus eu tenho tudo isso, convivo bem com meu marido, meus familiares [...]. (S12)

A efetiva rede de apoio ao idoso perpassa pela questão fundamental da comunicação, compreender o que se diz e se fazer compreensível. Nessa relação estão implícitos fatores como idade, aspecto sociocultural e condições físico-cognitivas.

Tendo em vista a promoção da saúde é interessante que ocorra uma reorganização na dinâmica familiar, de modo que toda a família repense sua alimentação a fim de torná-la mais saudável e contribuindo, por consequência, para adaptação do portador de DM.

Gosto de sentar com meus irmãos num churrasco, mas carne gorda eu não como [...]. (S2)

A adesão da pessoa diabética ao plano terapêutico tem uma estreita relação com o apoio familiar. Segundo a OMS, o adequado suporte social ao idoso relaciona-se à redução de morbimortalidade e disfunções psicológicas, bem como ao incremento da saúde e bem-estar geral. 
Entretanto, um estudo realizado em Botucatu constatou que, quanto mais a família controla o portador de DM, mais este questiona sua autonomia e mais dificuldade encontra para seguir as recomendações para controle da glicemia, desta maneira, retroalimentando o ciclo, que se torna vicioso. ${ }^{14}$ Logo, deve-se avaliar a qualidade dos vínculos familiares.

Percebe-se também que os idosos que residem sozinhos conferem especial significância aos animais de estimação, considerando-os "companheiros".

Moro sozinha, eu e meu cachorro, faço todo meu serviço, [...] minha filha mora perto de mim. (S4)

Eu sou sozinho, não tenho parente, não tenho nada, mais vivo com o que mais amo, meus quatro cachorros. (S8)

Para Schütz, a ação poder ser clara ou estar encoberta, mas nunca é divorciada do mundo, este horizonte é permeado pelas "relações familiares", no sentido de proximidade. ${ }^{10}$ Deste modo, este estudo mostrou que os vínculos afetivos foram considerados essenciais pelos idosos como garantia de uma qualidade de vida melhor.

\section{É viver com bem-estar emocional, espiritual e moral}

Outro aspecto preponderante nos depoimentos é o bemestar emocional-espiritual. Observa-se que, para eles, a qualidade de vida relaciona-se aos valores e às atitudes morais adotadas no decorrer de suas vidas, por vezes influenciadas pela religiosidade.

\section{[...] Vivo com paz no coração. (S1) Eu acho que éa pessoa andar na paz de Deus, não ter encrenca com ninguém, não maltratar ninguém. (S5) É paz de espírito, consciência tranquila. (S8)}

Um estudo realizado com 147 idosos em Portugal revela que estes apresentam disposição para a gratidão e amor compassivo pelo próximo e por toda a humanidade..$^{15}$

Para Schütz, o mundo social está intimamente relacionado com os outros e se organiza em campos, tais como o mundo dos contemporâneos, que convivem no meu tempo e espaço, o mundo dos que me antecederam e o mundo dos que me sucederão. Nestas relações, há graus diversos de intimidades e de anonimato. ${ }^{10}$

\section{[...] tenho minha religião, participo e frequento com todos os colegas meus, que inclusive me visitam em casa e estão sempre comigo. (S7 Eu acho que é a pessoa andar na paz de Deus [...]. (S5)}

A participação religiosa torna-se importante pelo apoio social receido ao frequentar a igreja, pela satisfação das relações sociais proporcionadas pela interação religiosa, como meio para ultrapassar a mortalidade, bem como pelo sentimento de uma relação íntima com Deus, que oferece a sensação de segurança e estimula os mecanismos de "coping" positivos. ${ }^{15}$

Acreditam ser importante respeitar o próximo e ter atitudes socialmente aceitas. A prática do bem junto aos semelhantes, saber ser honesto, não dever nada a ninguém, foi evidenciada nas falas.

É não interferir na vida de ninguém e não deixar que interfiram na sua. (S8)

Eu acho que é a pessoa ser honesta, não mexer no que é dos outros. (S5)

Não tenho quem me ajude, mas não também tenho quem me aborreça [...]. (S4)

A relação com nossos contemporâneos pode ser de dois modos, distante ou próxima em uma relação "face-aface", e esta é uma relação privilegiada, pois permite a intersubjetividade dos sujeitos, o intercâmbio de pontos de vistas e uma comunicação efetiva. É possível neste tipo de relação estabelecer vínculos afetivos e construções de rede de apoio. ${ }^{9}$

Logo o bem-estar emocional, espiritual e moral são essenciais para o idoso diabético viver com tranquilidade.

\section{É ter que viver com o diabetes mellitus}

Embora em alguns depoimentos a percepção do idoso em relação à sua própria qualidade de vida tenha sido negativa, a análise dos discursos mostrou que, em geral, este grupo acredita ter uma qualidade de vida relativamente boa.

Sou cega há nove anos, acho que foi o diabetes, mas sempre trabalhei, sempre fiz tudo. Eu tenho uma vida boa. (S1) Acho que, em geral, em relação aos dependentes da Bolsa-Familia do Lula, minha vida é muito boa. (S8)

Isso nos remete a pensar que o conceito clássico de Saúde da OMS mostra-se inadequado para descrever o universo de saúde dos idosos, já que a ausência de enfermidades é privilégio de poucos, e o completo bem-estar pode ser atingido por muitos, independente da presença de doenças. ${ }^{13}$

Corroborando nossos achados, uma pesquisa realizada na Colômbia encontrou que $16,6 \%$ dos idosos portadores de diabetes consideraram sua saúde como excelente/muito boa, e 59,7\%, como boa, a despeito das incapacidades e limitações que a doença pode causar. Esclarece ainda que a maioria dos idosos constituinte da amostra $(76,2 \%)$ não relatou complicações decorrentes da doença, as quais poderiam comprometer a autoavaliação do estado de saúde. ${ }^{6}$

A maioria dos idosos entrevistados referiu ter uma vida normal, sem grandes complicações, apesar de ter que 
Idosos portadores de diabetes: qualidade de vida

conviver com o DM e as restrições que este impõe, o que pode ser observado pelas falas:

Minha vida é normal, [...]. (S4) [...] Diminuo a carne, o açúcar, a cervejinha, mas não uso muito açúcar mesmo [...] vivo minha vida tranquilo assim. (S7) [...] Controlo bem o diabetes, peguei o diabetes na menopausa, mas nunca teve nada que eu achasse ruim não, estou controlando direitinho, só isso mesmo. (S12)

A literatura apresenta o DM compensado como fator positivo na busca pela melhoria da qualidade de vida. Um idoso com uma ou mais doenças crônicas pode ser considerado saudável, quando comparado com idoso com as mesmas doenças, porém sem controle, com sequelas decorrentes e incapacidades associadas. ${ }^{16}$

0 idoso diabético vive uma contradição, relata que a doença não interfere em seu viver, no entanto, esclarece que tem certos "cuidados". Sente-se sem opções ao ter que aceitar as restrições impostas pela sua condição crônica. Desta maneira, percebe-se também uma certa resignação.

O diabetes não me aborrece em nada, só que eu tenho cuidado, evito refrigerante, frituras, essas coisas eu não como não. (S4) Proíbe isso, proíbe aquilo [...] não pode tomar uma coisa meio doce quejá atrapalha [...]. (S5)

A doença crônica, muitas vezes, só é percebida por seus portadores quando apresenta sintomatologia específica associada; logo, quando têm essa apresentação, sentem-se saudáveis, consequentemente não seguem 0 tratamento recomendado.

Ah, eu como bem, nossa! Eu adoro as coisas que eu sei que são perigosas, eu sempre faço doce, e não faço aquele regime verdadeiro que eu sei que tenho eu fazer, não posso dizer que eu faço porque eu não faço. (S2) Açúcar? Sou um formigão. Não deixo de comer doce mesmo. Cigarro, fumo cinco por noite, sempre após o jantar. Álcool, muito pouco. Não sou abstêmio, mas também não sou alcoólatra. (S8)

Entretanto, convém lembrar que o idoso diabético pode apresentar menor sensibilidade para distinguir sabores, e tem tendência para ingerir maior quantidade de gordura e carboidrato, escolhidos pelo seu baixo custo, rápido preparo e fácil ingestão. ${ }^{17}$

Nas entrelinhas dos depoimentos, é evidente que a restrição aos alimentos cerceia também ações da própria vida, desta maneira podendo dificultar a proposta terapêutica instituída.
Mas pra mim qualidade de vida é você poder comer tudo aquilo que gosta, não ter que ficar regulando, segurando [...] não pode comer isso, não pode comer aquilo, né? É dificil pra quem tem diabetes, eu gosto muito de cozinha, massa, pão, não pode [...](S2)

0 tratamento e o acompanhamento do diabetes envolvem mudanças de hábito e monitoramento domiciliar da glicemia. Devem ser uma ação multiprofissional com o envolvimento familiar. Os profissionais da saúde precisam estar atentos para o fato de que o idoso pode apresentar alterações relacionadas ao processo de envelhecimento, as quais aumentam as dificuldades de adesão ao tratamento, tais como alterações cognitiva, sensorial, cutânea, gustativaolfatório. ${ }^{16}$ As ações por parte dos profissionais devem ser somadas com as dos familiares, devem ter objetivos comuns.

Desse modo, constata-se, por meio dos depoimentos deste grupo de idosos, que é necessário conviver com o diabetes e aceitar as mudanças de hábito, embora não deva determinar toda sua existência.

Percebe-se que viver com DM é complexo, não apresenta linearidade. Trata-se de um processo que interage dinamicamente com diversas facetas do viver cotidiano, influenciando e sendo influenciado por inúmeras relações. Evidencia-se um viver dinâmico com múltiplas possibilidades, mas que se pode caminhar para a manutenção ou conquista do viver com qualidade.

\section{CONSIDERAÇÕES FINAIS}

0 estudo possibilitou o desvelamento de algumas facetas relevantes quanto ao significado de qualidade de vida para idosos portadores de DM2. As convergências analisadas evidenciam que, para este grupo de idosos diabéticos, a qualidade de vida está atrelada à saúde física, independência na vida diária e econômica, integração social, suporte familiar e saúde mental-espiritual.

Em relação ao conviver com DM constata-se que a restrição alimentar é o ponto de maior repercussão. No entanto, para estes idosos, embora a doença faça parte do seu viver, ela não se configura como o centro de sua existência. Com isso, conseguem encontrar forças para vencer as restrições e as possíveis complicações decorrentes de DM2.

Afinal, fica claro que o conviver com diabetes não se limita à adesão ou não ao tratamento, mas à possibilidade de ampliar o diálogo e negociação dentro de uma ética de respeito às diferenças que, expressas, poderão ser discutidas em busca de autonomia e independência no cuidado de saúde, com corresponsabilização.

Este estudo, não sendo estatístico, não pretende generalizar, mas descrever e compreender o significado de qualidade de vida para idosos diabéticos, contextualizado 
no local e tempo em que se desenvolveu, pois entende-se que esta compreensão traz subsídios para implementar ações em prol da busca do bem-estar na terceira idade.

\section{REFERÊNCIAS}

1.Veras R. Envelhecimento populacional e as informações da saúde do PNAD: demandas e desafios contemporâneos. Cad Saude Publica. 2007; 23(10): 2463-66.

2.Moreira MD, Caldas CP. 0 cuidador e a saúde da pessoa idosa. Esc Anna Nery . 2007; 11 (3): 520 - 25.

3.Francisco PMSB, Belon AP, Barros MBA, Carandina L, Alves MCGP, Goldbaum M, et al. Diabetes auto-referido em idosos: prevalência, fatores associados e práticas de controle. Cad Saude Publica. 2010; 26(1):17584.

4.Figueiredo DM, Rabelo FLA. Diabetes Insipidus: principais aspectos e análise comparativa com diabetes mellitus. Semina: Ciências Biológicas e da Saúde. 200930 (2): 155-162.

5.Ministério da Saúde (BR). Secretaria de Atenção à Saúde. Departamento de Atenção Básica. Diabetes Mellitus. Brasília: DF; 2006.

6.Lazcano-Ortiz M, Salazar-González BC. Adaptación en pacientes com diabetes Mellitus tipo 2 según modelo de Roy. Aquichan. 2009; 9(3): 236-45.

7.Jóia LC, Ruiz T, Donalisio MR. Condições associadas ao grau de satisfação com a vida entre a população de idosos. Rev Saude Publica. 2007; 41(1): 131-38.

8.Vecchia RD, Ruiz T, Bocchi SCM, Corrente JE. Qualidade de vida na terceira idade: um conceito subjetivo. Rev Bras Epidemiol. 2005 8(3): 246-52.

9.Wagner H. Fenomenologia e relações sociais, textos escolhidos de Alfred Schütz. Rio de Janeiro(RJ):Zahar; 1979

10.Capalbo C. Metodologia das ciências sociais: a fenomenologia de Alfred Schütz. $2^{\mathrm{a}}$ ed. Londrina(PR): UEL; 1998.

11.Parga Nina L. Estudos das informações não estruturadas do ENDEF e sua interpretação com os dados qualificados. Rio de Janeiro(RJ): IBGF; 1956. p. 215.

12.Carvalhais Neto N. Envelhecimento bem-sucedido e envelhecimento com fragilidade. In. Ramos LR, Toniolo Neto J, organizadores. Geriatria e gerontologia. Barueri (SP): Manole; 2005. p. 9-25.

13.Ferreira FS. Qualidade de vida relacionada à saúde dos indivíduos atendidos por uma equipe de saúde da família do município de Uberaba [dissertação de mestrado]. Ribeirão Preto (SP): Escola de Enfermagem de Ribeirão Preto,Universidade de São Paulo; 2008.
14.Caixeta CC. As relações familiares e o processo de adoecimento em diabetes tipo Il [dissertação] Ribeirão Preto (SP): Escola de Enfermagem de Ribeirão Preto, Universidade de São Paulo; 2007.

15.Menezes APLF. A propensão para o perdão na pessoa idosa [Internet] . Cidade do Porto (PT): Faculdade de Psicologia e de Ciências da Educação, Universidade do Porto. [citado 2010 abr 28]. Disponível em: http:// repositorio-aberto.up.pt/handle/10216/13640.

16.Nasri F, Sá JR. Diabtes Mellitus. In: Ramos LR, Toniolo Neto J, organizadores. Geriatria e gerontologia. Barueri (SP): Manole; 2005. p. 77-94.

17. Oviedo AD. A pessoa com diabetes: do enfoque terapêutico ao existencial [tese]. Ribeirão Preto (SP): Escola de Enfermagem de Ribeirão Preto, Universidade de São Paulo; 2007. 